Mon. Not. R. Astron. Soc. 000, 12 (1997) Printed 1 January 2018

\title{
On the galactic disc age-metallicity relation
}

\author{
Giovanni Carraro ${ }^{1,2}$, Yuen Keong $\mathrm{Ng}^{3}$ and Laura Portinari ${ }^{1,4}$ \\ 1 Department of Astronomy, Vicolo dell'Osservatorio 5, I-35122 Padova, Italy \\ 2 SISSA-ISAS, Via Beirut 2-4, I-34014 Trieste, Italy \\ 3 Osservatorio Astronomico di Padova, Vicolo dell'Osservatorio 5, I-35122 Padova, Italy \\ 4 Nordita, Blegdamsvej 17, DK-2100 Copenhagen E, Denmark \\ E-mail: carraro, yuen,portinari@astrpd.pd.astro.it
}

submitted

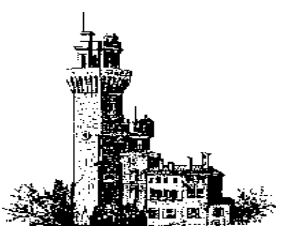

\begin{abstract}
A comparison is made between the age-metallicity relation obtained from four different types of studies: F and G stars in the Solar Neighbourhood, analysis of open clusters, galactic structure studies with the stellar population synthesis technique, and chemical evolution models. Metallicities of open clusters are corrected for the effects of the radial

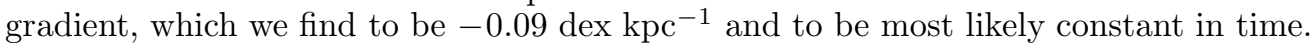
We do not correct for the vertical gradient, since its existence and value are not firmly established.

Stars and clusters trace a similar age-metallicity relation, showing an excess of rather metal-rich objects in the age range 5-9 Gyr. Galactic structure studies tend to give a more metal-poor relation than chemical evolution models. Both relations do not explain the presence of old, relatively metal-rich stars and clusters. This might be due to uncertainties in the ages of the local stars, or to pre-enrichment of the disc with material from the bulge, possibly as a result of a merger event in the early phases of the formation of our Galaxy.
\end{abstract}

Key words: Open clusters - Galaxy: abundances, gradients, chemical evolution, general, structure

\section{INTRODUCTION}

The age-metallicity relation (AMR) for nearby stars is a record of the progressive chemical enrichment of the starforming local interstellar medium during the evolution of the galactic disc, and so provides useful clues about the star formation and chemical evolution history of the local environment. Metallicity is usually identified with the $[\mathrm{Fe} / \mathrm{H}]$ ratio. Oxygen would actually be a better tracer and "chronometer" of metal enrichment (Wheeler et al. 1989), because it is the most abundant metal and it is produced on the well-defined and short time-scale of type II SNæ. On the contrary, iron is released by various sources (both type II and type Ia SNæ) with rather different time-scales. Anyway, metallicity is generally determined by the $[\mathrm{Fe} / \mathrm{H}]$ ratio, since the $[\mathrm{O} / \mathrm{H}]$ ratio is much more difficult to measure in stellar atmospheres.

In the Solar Neighbourhood the metallicity of the stars can be studied in high detail (Edvardsson et al. 1993, hereafter Edv93ea), but the results are liable to large errors in the individual determinations of the age. $\mathrm{Ng} \&$ Bertelli (1998, hereafter NB98) demonstrated that the errors in the age are mainly due to the large uncertainties in the individual distances. Distances need to be known to a $5 \%$ accuracy to get reliable ages. In this respect, the ages and metallicities of clusters are more reliable, since one is dealing with a group of stars and therefore the result is less susceptible to individual errors.

An AMR can also be obtained from star counts studies, based on the population synthesis technique (Bertelli et al. 1995, 1996; Ng et al. 1995, 1996, 1997). In such studies all the stars along the line of sight are considered. The disc is sampled with respect to age and metallicity in layers with specific effective thicknesses. In this way indications are obtained for the disc's chemical evolution.

The aim of this paper is to compare the AMR obtained from various methods and to critically discuss the probable causes for the differences found. In Sect. 2 we start with a general overview of the various AMRs and improve them when possible. In Sect. 3 the relations are compared with each other and probable causes for any discrepancy are outlined. The results are finally summarized in Sect. 4 .

\section{AGE-METALLICITY RELATION}

In this section we describe the AMRs considered for this study. We start with the AMR obtained from stars in the Solar Neighbourhood. Then we consider the AMR obtained from open clusters, by improving the results from Carraro \& Chiosi (1994a, hereafter CC94a) with a larger 




Figure 1. AMR for nearby stars by Meusinger et al. (1991; triangles) and Edvardsson et al. (1993, open squares and open circles).

cluster sample. We continue with the description of the AMR obtained from star counts analysis based on the stellar population synthesis technique. Finally, we discuss the AMR obtained from chemical evolution models.

\subsection{The Solar Neighbourhood}

Twarog (1980) first showed that nearby stars display an AMR, by applying uvby- $\beta$ photometry and Yale isochrones to get the metallicity and the age for two wide samples of stars. Since single data showed a remarkable dispersion, agebins and average metallicity per bin were used to deduce the local AMR. The metallicity turned out to increase sensitively during disc evolution, rapidly from 13 to 5 Gyr ago, and more slowly onwards.

Twarog's data were later re-examined by means of different photometric calibrations with the Vandenberg (1983, 1985) isochrones, resulting in discordant AMRs.

Carlberg et al. (1985) limited themselves to only one of the two samples from Twarog, hereby excluding around fifty of the lowest metallicity stars. They obtained a relatively high average metallicity for old stars and a shallower slope of the AMR in the early phases, suggesting that the disc evolution started with a high initial metallicity.

On the other hand, Meusinger et al. (1991) re-examined both samples from Twarog with Vandenberg's isochrones and with a new calibration for the metallicity index and improved model atmospheres. The resulting AMR was closer to the one obtained by Twarog and had a considerable slope toward old ages. This relation is displayed in Fig. 11.

A new estimate of the local AMR, based on a sample of 189 nearby $\mathrm{F}$ and $\mathrm{G}$ dwarfs, was performed by Edv93ea. The metallicity is derived from the analysis of high resolution spectra with theoretical LTE model atmospheres. The ages were determined from fits of the photometric data with Vandenberg (1985) isochrones. The resulting binned AMR is in good agreement, within the large dispersion, with that by Meusinger et al. Figure 1 shows the averaged, binned AMR (open squares) from Edv93ea together with the individual data points.

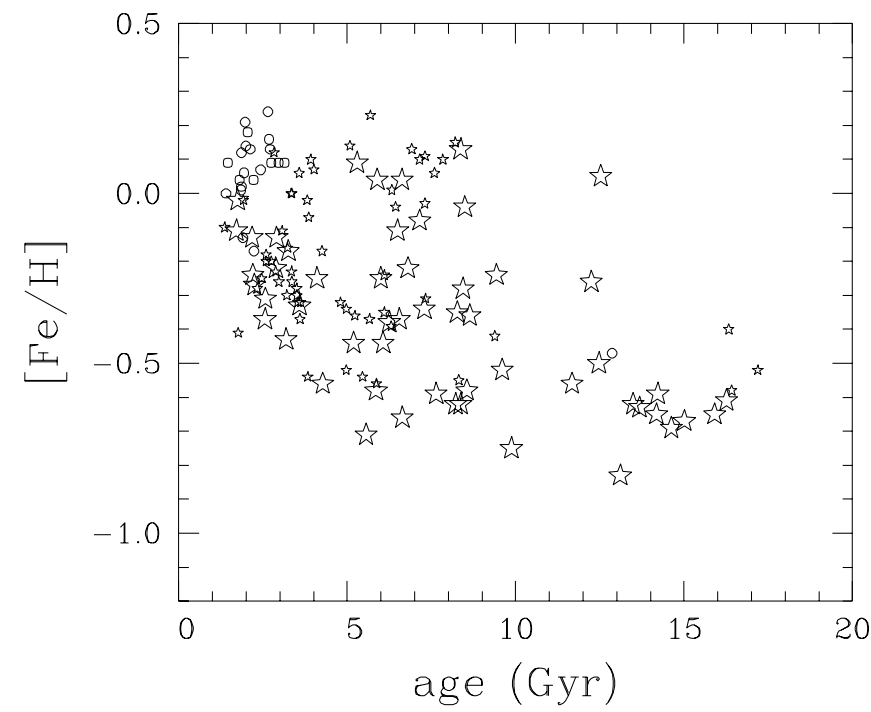

Figure 2. The AMR for nearby stars by Ng \& Bertelli (1997). The ages are computed with the distances obtained from the Hipparcos parallax (ESA 1997). The open circles indicate star on or near the main sequence with an uncertainty in the age less than $12 \%$. Open stars are used for giant branch stars. The uncertainty in the age is less than $12 \%$ for the large symbols and greater than $12 \%$ for the small symbols.

The Edv93ea dataset was re-analysed by NB98 by means of Bertelli et al. (1994) isochrones, based on the latest opacity tables. Near the main sequence, high age isochrones are packed closely together and the deduced ages have considerable uncertainties. The situation changes if a star is on the giant branch: its evolution is relatively fast and the uncertainty in the age becomes considerably smaller. For the determination of the ages, NB98 also took into account the uncertainties on the effective temperature, metallicity and distance of each star; the distances of the stars are obtained from the Hipparcos (ESA 1997) parallaxes. In this way, reliable ages were obtained for the stars displayed with big symbols in Fig. A. The resulting AMR has a small, but distinct slope of $\sim 0.07 \mathrm{dex} / \mathrm{Gyr}$ when the stars older than $10 \mathrm{Gyr}$ are considered. However, NB98 suspect that the ages for $t>10$ Gyr might be overestimated ( $\sim 2$ Gyr), due to an improper selection of the isochrones for these stars, which are over-abundant in $\alpha$-elements. It is noteworthy that this relation is essentially not different from the relation one gets when, for the stars with reliable ages, the ages from Edv93ea are adopted; we refer to NB98 for additional details.

However, the most striking feature of the results mentioned above is the huge scatter around the average trend, which makes the correlation between age and metallicity rather weak, especially for stars with $t<10$ Gyr. Such a spread in the data must be in part intrinsic, and many possible causes have been suggested: orbital diffusion of stars coupled with radial metallicity gradients, local inhomogeneities in the star-forming gas, inhomogeneous accretion of infalling external gas, overlapping of different galactic sub-structures, each with its own specific AMR. All the mentioned effects may actually contribute to produce the observed scatter, and the new challenge for chemical models is nowadays to explain such a large spread in the local AMR (see Sect.2.4). 
On the galactic disc age-metallicity relation

Table 1. Sample of 37 clusters with: ages determined by the synthetic CMDs technique ( $2^{\text {nd }}$ column), spectroscopic metallicities in the Boston scale (Friel \& Janes 1993, Friel 1995; $3^{\text {rd }}$ column) and corresponding errors ( $4^{\text {th }}$ column), height above the galactic plane ( $5^{\text {th }}$ column) and galactocentric distance ( $6^{\text {th }}$ column).

\begin{tabular}{|c|c|c|c|c|c|}
\hline Cluster & age(Gyr) & {$[\mathrm{Fe} / \mathrm{H}]$} & r.m.s. & $z(\mathrm{pc})$ & $\mathrm{R}(\mathrm{kpc})^{\dagger}$ \\
\hline Berkeley 17 & 9.00 & -0.29 & 0.13 & 170 & 11.2 \\
\hline Berkeley 19 & 3.80 & -0.50 & 0.10 & 300 & 13.3 \\
\hline Berkeley 20 & 3.00 & -0.75 & 0.21 & 2100 & 16.0 \\
\hline Berkeley 21 & 3.10 & -0.97 & 0.10 & 260 & 14.5 \\
\hline Berkeley 31 & 4.00 & -0.50 & 0.16 & 430 & 12.7 \\
\hline Berkeley 32 & 3.00 & -0.58 & 0.10 & 300 & 12.0 \\
\hline Berkeley 39 & 6.50 & -0.31 & 0.08 & 705 & 11.7 \\
\hline Cr 261 & 7.00 & -0.14 & 0.14 & 420 & 7.6 \\
\hline IC 166 & 0.85 & -0.32 & 0.20 & 10 & 10.7 \\
\hline IC 4651 & 1.60 & -0.16 & 0.05 & 100 & 7.8 \\
\hline King 5 & 0.80 & -0.38 & 0.20 & 180 & 10.5 \\
\hline King 11 & 6.00 & -0.36 & 0.14 & 460 & 10.5 \\
\hline M 67 & 4.80 & -0.09 & 0.07 & 415 & 9.1 \\
\hline Melotte 66 & 5.46 & -0.51 & 0.11 & 710 & 9.4 \\
\hline NGC 188 & 7.50 & -0.05 & 0.11 & 570 & 9.3 \\
\hline NGC 752 & 1.50 & -0.16 & 0.05 & 145 & 8.7 \\
\hline NGC 1193 & 5.00 & -0.50 & 0.18 & 1020 & 12.7 \\
\hline NGC 1245 & 0.80 & +0.14 & 0.10 & 460 & 11.1 \\
\hline NGC 1817 & 0.80 & -0.39 & 0.04 & 410 & 10.3 \\
\hline NGC 2141 & 2.50 & -0.39 & 0.11 & 430 & 12.6 \\
\hline NGC 2158 & 1.42 & -0.23 & 0.07 & 95 & 11.6 \\
\hline NGC 2204 & 1.74 & -0.58 & 0.10 & 1200 & 11.8 \\
\hline NGC 2243 & 4.50 & -0.56 & 0.17 & 1260 & 11.1 \\
\hline NGC 2420 & 2.10 & -0.42 & 0.07 & 655 & 10.3 \\
\hline NGC 2477 & 0.60 & -0.05 & 0.11 & 135 & 9.0 \\
\hline NGC 2506 & 1.90 & -0.52 & 0.07 & 460 & 10.4 \\
\hline NGC 2660 & 0.70 & +0.06 & 0.10 & 152 & 9.2 \\
\hline NGC 3680 & 1.80 & -0.16 & 0.05 & 230 & 8.3 \\
\hline NGC 3960 & 0.60 & -0.34 & 0.08 & 180 & 8.0 \\
\hline NGC 5822 & 0.45 & -0.21 & 0.12 & 45 & 7.9 \\
\hline NGC 6791 & 8.00 & +0.19 & 0.19 & 1010 & 8.4 \\
\hline NGC 6819 & 2.05 & +0.05 & 0.11 & 310 & 8.2 \\
\hline NGC 6939 & 1.40 & -0.11 & 0.10 & 255 & 8.7 \\
\hline NGC 6940 & 0.60 & +0.04 & 0.10 & 100 & 8.3 \\
\hline NGC 7142 & 4.90 & 0.00 & 0.06 & 495 & 9.7 \\
\hline NGC 7789 & 1.35 & -0.26 & 0.06 & 165 & 9.4 \\
\hline Tombaugh 2 & 1.75 & -0.70 & 0.18 & 1030 & 15.6 \\
\hline
\end{tabular}

$\dagger \quad \mathrm{R}$ is computed with $R_{\odot}=8.5 \mathrm{kpc}$ for the distance of the Sun to the galactic centre for consistency with previous work.

\subsection{Open clusters}

\subsubsection{Sample}

Most of the open clusters considered in this paper (Table 1) are taken from the compilation presented by CC94a. We selected from that sample all the clusters for which a homogeneous metallicity determination was available: all the metallicities in Table 1 are in fact obtained spectroscopically and they are in the Boston scale (Friel \& Janes 1993, Friel 1995). Therefore, with respect to CC94a we do not consider here NGC 6603, IC 1311, NGC 7704, King 2 and AM 2, since for these cluster the lack of a homogeneous metallicity estimate prevents us from obtaining their age with the same method as for all the other clusters.

In addition, the CC94a sample has been updated including here Berkeley 17 (Kaluzny 1994), Berkeley 20, Berkeley 31 and King 5 (Phelps et al. 1994), Collinder 261 (Mazur et al. 1995), and NGC 1245 (Carraro \& Patat 1994). Table 1 lists our updated compilation for 37 clusters.

In CC94a, the ages of ten clusters were determined with stellar isochrones using the synthetic Colour-magnitude diagrams (CMDs) technique (Chiosi et al. 1989). This sample defined a relation between age and $\Delta \mathrm{V}$ (cf. Cannon 1970, Cameron 1985, Barbaro \& Pigatto 1984, AnthonyTwarog \& Twarog 1985). The ages of the remaining clusters were obtained through interpolation of this relation, only in a few cases an extrapolation was applied. von Hippel et al. (1995) noticed for one cluster a discrepancy between the extrapolated $\Delta \mathrm{V}$ age with the age determined from the white dwarf cooling sequence. This however does not imply, as concluded by von Hippel et al. (1995), that the isochrone ages are in error. It mainly demonstrates that one has to be cautious with extrapolations.

In this paper, the ages for all the clusters in Table 1 have been determined by fits with the Bertelli et al. (1994) isochrones using the synthetic CMDs technique, following the procedure described by Carraro \& Chiosi (1994a, 1995). We found for Berkeley 17 and Collinder 261 an age sensitively different from recent studies (Phelps 1997 and Gozzoli et al. 1996 respectively). In the case of Berkeley 17 the different value can be ascribed to the use of different datasets and different techniques (simple isochrones fitting against synthetic CMDs). The same partly holds for Collinder 261. We used a different dataset and a slightly different method. Gozzoli et al. 1996 derived the age from evolutionary tracks (Fagotto et al. 1994), while we determined the age from suitably interpolated isochrones (Bertelli et al. 1994). Finally, we performed simulations with synthetic CMDs and used the capability of our code to interpolate between isochrones of different metallicities, adopting for the simulations a metallicity $Z$ corresponding to the observational $[\mathrm{Fe} / \mathrm{H}]$.

The main advantage of this compilation is the homogeneity of the sample: ages, metallicities and positions in the galactic disc are all obtained in the same fashion. This homogeneity is not guaranteed when using larger samples as in Friel (1995), Piatti et al. (1995, hereafter P95ea) or Twarog et al. (1997). In addition, completeness is a crucial ingredient in order to explore possible relations between the cluster properties and their position in the galactic disc. However, it is not possible to gather a complete sample for the old, open clusters in the galactic disc, because of strong selection effects mainly related to the past dynamical history of the galactic disc. Many old clusters have probably been disrupted (van den Bergh \& McClure 1980; Friel 1995). Taken this limitation into account, we used a statistical approach to find correlations between the clusters' fundamental parameters, i.e. age, metal abundance and position inside the galactic disc.

\subsubsection{Multivariate analysis}

The search for correlations has been realized by means of Multivariate Data Analysis (Murtagh \& Heck 1987) with the SPSS package (Nie et al. 1975). In particular, we performed the so-called Principal Component Analysis (PCA), which is designed to find the number of independent parameters characterizing multivariate data. This technique is widely 

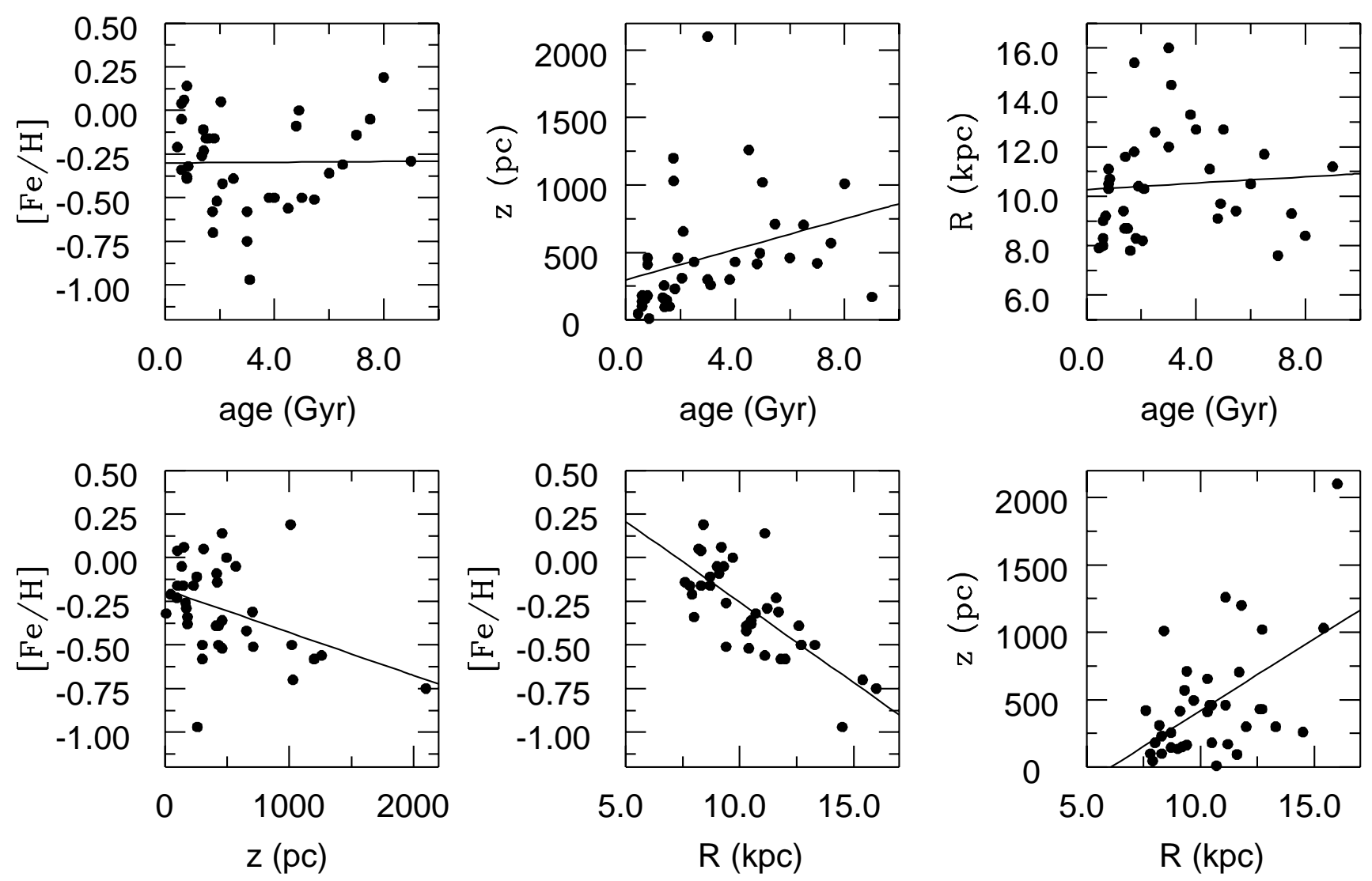

Figure 3. Relation between the fundamental parameters of the open cluster sample given in Table 1, see Sects. $2.2 .2 \& 2.2 .3$ for details. Solid lines are the projections of the first principal component: $\left([\mathrm{Fe} / \mathrm{H}]-t_{9}\right),\left(z-t_{9}\right),\left(\mathrm{R}-t_{9}\right),([\mathrm{Fe} / \mathrm{H}]-z),([\mathrm{Fe} / \mathrm{H}]-\mathrm{R})$, and $(z-\mathrm{R})$.

used in astronomy; for applications to other astronomical problems we refer to Murtagh \& Heck.

The crux of the method is to search for suitable linear coordinate transformations, which are by definition orthogonal, and to diagonalize the covariance matrix from an original set of variables $\left(x_{1}, x_{2}, \ldots x_{n}\right)$ to a new set of variables $\left(\psi_{1}, \psi_{2}, \ldots \psi_{n}\right)$ with a zero mean value:

$$
\psi_{i}=\sum_{k=1}^{n} l_{i k} x_{k},
$$

where $l_{i k}$ are the coefficients of the transformation. The properties of the transformation are such, that the eigenvalues $\lambda_{i}$ of the matrix $\left\langle\psi_{i} \psi_{j}>\right.$ are the variances of the data in the direction of the principal component. The corresponding eigenvectors are then used to build the new linear combinations from the initial parameters. By convention, the first principal component corresponds to the largest eigenvalue. As a consequence, the first principal component is a minimum distance fit to a line in the space of the original parameters. The same holds for a possible second, third and so on principal component.

\subsubsection{Principal Components}

In our case the original space of parameters is fourdimensional. The parameters are the age, the metallicity, the $z$-coordinate and the radial distance $\mathrm{R}$ from the galactic centre ( $\mathrm{R}$ is computed with $R_{\odot}=8.5 \mathrm{kpc}$, where $R_{\odot}$ is the
Table 2. Principal components $\left(\psi_{1}, \psi_{2}\right)$ and eigenvalues for the open cluster sample listed in table 1 , together with the values $\left(\psi_{1 p}, \psi_{2 p}\right)$ for the Piatti et al. (1995) sample.

\begin{tabular}{lcrcrcc}
\hline$l_{i j}$ & age & {$[\mathrm{Fe} / \mathrm{H}]$} & $z$ & \multicolumn{1}{c}{$\mathrm{R}$} & $\lambda$ & variance $(\%)$ \\
\hline$\psi_{1}$ & 0.201 & -0.849 & 0.755 & 0.914 & 2.167 & 54.2 \\
$\psi_{2}$ & 0.938 & 0.284 & 0.256 & -0.153 & 1.049 & 26.2 \\
& & & & & & \\
$\psi_{1 p}$ & 0.624 & -0.890 & -0.331 & 0.782 & 1.903 & 47.6 \\
$\psi_{2 p}$ & 0.619 & 0.100 & 0.873 & -0.011 & 1.155 & 28.9 \\
\hline
\end{tabular}

distance of the Sun to the galactic centre). The straight application of PCA leads to the identification of two principal components shown in Table 2.

The first principal component $\psi_{1}$ gathers more than $50 \%$ of the variance. Its coefficients are an indication for the degree of dependence it has to the original parameters. It appears from the first component that the clusters occupy a threedimensional sub-space inside the four-dimensional parameters space: a sort of strongly elongated cigar whose main axis is almost parallel to the age axis. Therefore, the old open clusters in Table 1 form a one-parameter family.

The projections of the first principal component on the six planes are: $\left([\mathrm{Fe} / \mathrm{H}]-t_{9}\right),\left(z-t_{9}\right),\left(\mathrm{R}-t_{9}\right),([\mathrm{Fe} / \mathrm{H}]-z)$, $([\mathrm{Fe} / \mathrm{H}]-\mathrm{R})$, and $(z-\mathrm{R})$. They correspond approximately to the usual regression fits between these pairs of variables. The results are shown in Fig. 3 . 

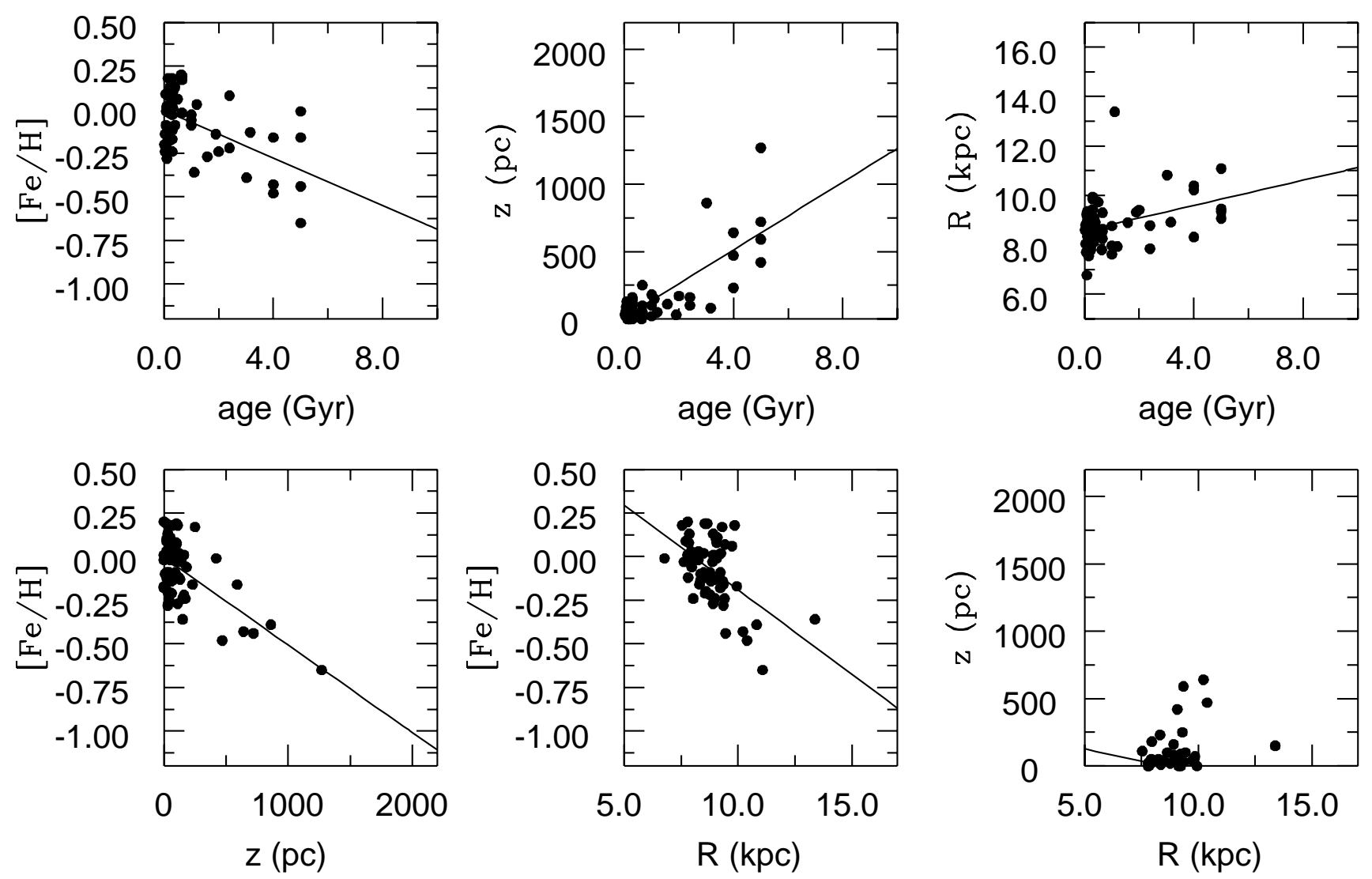

Figure 4. Relation between the fundamental parameters of the sample of open clusters from Piatti et al. (1995), see Sects. 2.2.2 \& 2.2.3. Solid lines are the projections of the first principal component: $\left([\mathrm{Fe} / \mathrm{H}]-t_{9}\right),\left(z-t_{9}\right),\left(\mathrm{R}-t_{9}\right),([\mathrm{Fe} / \mathrm{H}]-z),([\mathrm{Fe} / \mathrm{H}]-\mathrm{R})$, and $(z-\mathrm{R})$.

A similar analysis is made for the P95ea data set. To be representative, a clusters sample has to be well distributed in age, metallicity and position. Their sample includes 62 clusters with homogeneous metallicities, derived from DDO photometry. The other parameters are non homogeneous, because only one metal-poor cluster (NGC 2243) with $z>1 \mathrm{kpc}$ above the galactic plane is included and in addition they did not consider NGC 6791, an old, metalrich cluster with $z=1 \mathrm{kpc}$. Moreover, about half of the sample (37 clusters) is younger than 1 Gyr and located at $z=100 \mathrm{pc}$. Therefore, homogeneity in age and position is not necessarily guaranteed in their cluster sample. In the PCA analysis we follow for consistency P95ea and do not consider NGC 6791 and Lo 807. The latter cluster is excluded, because it lacks an age estimate. Concerning $\mathrm{Ru}$ 46 one has to keep in mind that Carraro \& Patat (1995) demonstrated that this is likely not an open cluster. Finally, the orbits calculated by P95ea are based on an out-of-date Galaxy model (see for instance Allen \& Santillan 1993) and in addition they did not correct the velocity components for Galactic differential rotation (Carraro \& Chiosi 1994b).

The results $\left(\psi_{1 p}, \psi_{2 p}\right)$ of the PCA are shown in Table 2, and the projection of the first component shown in Fig. 1 are in the same six planes as in Fig. 3. The lower eigenvalue and variance of the first principal component for the P95ea sample with respect to our sample is an indication that their dataset is less homogeneous.

\subsection{Stellar population synthesis}

The stellar population synthesis technique is used to generate synthetic Hertzsprung-Russell diagrams (HRDs) from either stellar evolutionary tracks or isochrones. It is a powerful tool to study resolved stellar populations. The evolutionary phases of a star are linked to each other through libraries with stellar evolutionary tracks. The ratio of the number of stars between different phases is directly related with the relative evolutionary time scale. This technique has been applied mainly to the analysis of stellar aggregates (Aparicio et al. 1990, Carraro et al. 1993, Aparicio \& Gallart 1995, Tosi et al. 1991, Vallenari et al. 1992).

The so-called HRD galactic software telescope (HRDGST) is developed to study the stellar populations in our Galaxy ( $\mathrm{Ng}$ 1994, $\mathrm{Ng}$ et al. 1995). The basis is formed by the latest evolutionary tracks calculated by the Padova group (Bertelli et al. 1994 and references cited therein). A smooth metallicity coverage is obtained through interpolation between the sets of tracks from low $(Z=0.0004)$ to high $(\mathrm{Z}=0.10)$ metallicity. Figure 5 shows a schematic diagram of the HRD-GST, see also $\mathrm{Ng}$ (1994) and $\mathrm{Ng}$ et al. (1995) for additional details.

Synthetic CMD are generated with a galactic model and ought to be comparable with those obtained from observations. The distribution of the stars along the line of sight is a complex mixture of populations. The ages, metallicities and spatial distributions of the stars from different populations contain a wealth of information about the structure, 


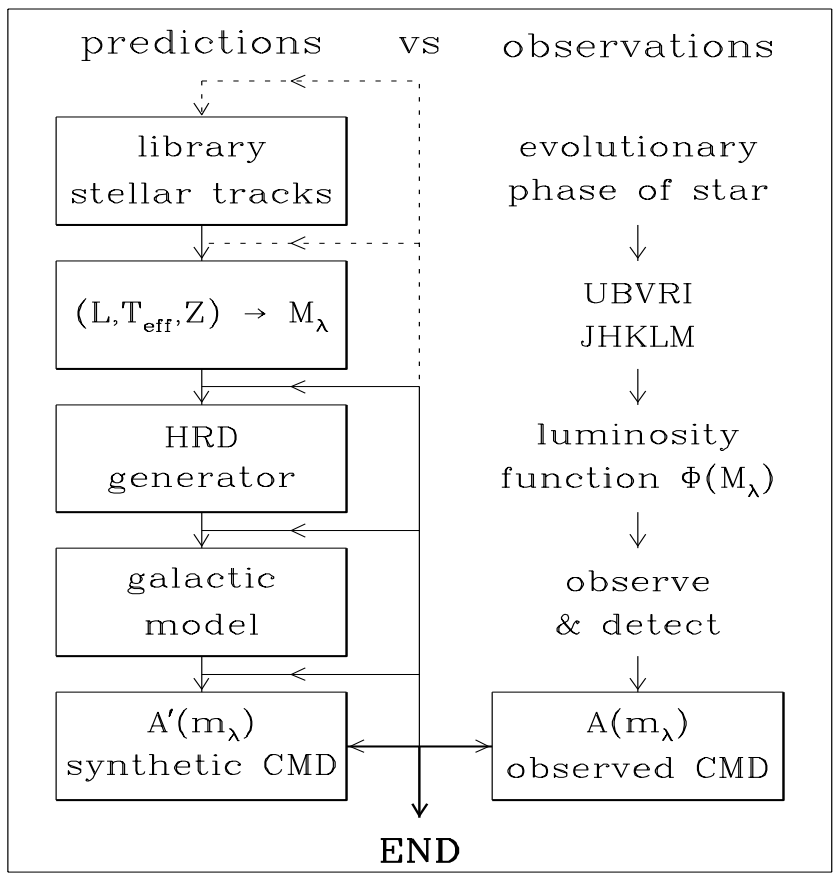

Figure 5. Schematic diagram of the HRD-GST. Input for the stellar population synthesis engine is the Padova library of stellar evolutionary tracks. The luminosities and effective temperature for each synthetic star of arbitrary metallicity is then transformed to an absolute magnitude in a photometric passband with the method outlined by Bressan et al. (1994) and Charlot et al. (1996). A synthetic HRD is generated, after specification of the stellar luminosity function through the initial mass function, the star formation rate and the age \& metallicity range. Synthetic stars from those diagrams are then 'observed' and 'detected' with the galactic model, through a Monte-Carlo technique. In this model the density distribution of each galactic component along the line of sight is specified. This results in a synthetic CMD of the field of interest. The synthetic CMD ought to be comparable with the observed CMD, when a realistic set of input parameters is used. If there is a marginal agreement then check the input for each step of the HRD-GST.

formation and evolution of our Galaxy.

The primary goal of the HRD-GST is to determine the interstellar extinction along the line of sight and to obtain constraints on the galactic structure and on the age-metallicity of the different stellar populations distinguished in our Galaxy. The results obtained thus far have been reported in various papers (Bertelli et al. 1995, 1996; $\mathrm{Ng}$ et al. $1995-1997)$. The disc is described by a mixture of subpopulations, each with its specific scale height and metallicity, respectively increasing and decreasing with age. The star formation of metal poor stars in the galactic disc commenced around 16-13 Gyr ago (Ng 1994, Ng et al. 1997). We adopted 13 Gyr for this paper. Figure 7 shows the AMR obtained with the HRD-GST together with data from stars in the Solar Neighbourhood and the line predicted by a chemical evolution model.

\subsection{Chemical evolution models}

The AMR for nearby stars is a standard constraint for modelling the chemical evolution of the Solar Neighbourhood, because it is a trace of the progressive storage of heavy elements in the star-forming local interstellar medium.

Most numerical or analytical chemical evolution models for the Solar Neighbourhood and for the galactic disc (Matteucci \& François 1989; Tosi 1988; Prantzos \& Aubert 1995; Ferrini et al. 1992, 1994; Pagel \& Tautvaisiene 1995; Timmes et al. 1995; Chiappini \& Matteucci 1997; Portinari et al. 1997; and references cited in those papers) follow the evolution of chemical abundances for a mixture of stars and gas, which is assumed to be chemically homogeneous in space. The disc is divided into concentric rings, each of which is treated as a homogeneous region. Therefore, such models are aimed at reproducing average features.

Many physical inputs of chemical models are rather poorly known and assumptions need to be made about the star formation rate (SFR), the initial mass function (IMF), the infall time-scale, and so forth. These quantities are usually parameterized and then calibrated on observational constraints. The predicted AMR for the Solar Neighbourhood is sensitive to the adopted SFR, infall time-scale and IMF. All chemical models are basically able to reproduce the observed average AMR (see, for instance, Fig. 7). As already mentioned in Sect. 2.1, recent studies of the AMR indicate the presence of a significant scatter with respect to the average trend. The scatter in metallicity for a given age is comparable to the overall average increase of metallicity from the early phases of disc evolution to the present time (Edv93ea). As a result, the average AMR no longer represents a tight constraint for chemical evolution models; the new challenge lies now in reproducing the dispersion of the data, rather than the average correlation. More complex chemical models are therefore required, which should include mechanisms inducing the observed scatter. We briefly summarize here various mechanisms that have been suggested.

a) Diffusion of stellar orbits allows stars to move away from their birth-places due to scattering by molecular clouds, by density waves in the disc or by infalling satellite galaxies. This phenomenon is revealed by the observed relation between the age and the velocity dispersion of disc stars (Wielen 1977, Wielen et al. 1992). Diffusion of stars from different birth-places into nearby orbits results in a spread of metallicities with respect to the expected "local" metallicity for a given age; François \& Matteucci (1993) discuss this effect in the framework of chemical evolution models. Edv93ea derived accurate kinematical data and explored the influence of stellar orbits on the scatter in the AMR: the scatter remains large, even for sub-samples with similar orbital properties. This might be an indication that another mechanism is required to explain the scatter, but the argument is still controversial (see Wielen et al. 1996).

b) Non-instantaneous mixing of stellar nucleosynthesis products in the surrounding gas allows for selfenrichment in molecular clouds and for local inhomogeneities (Malinie et al. 1993). Pilyugin \& Edmunds (1996) and van den Hoek\&de Jong (1997) combined selfenrichment due to sequential star formation with episodic infall of relatively metal-poor gas, triggering star formation on time-scales shorter than the time mixing takes to smear out chemical inhomogeneities. Both sequential star formation and infall are observed to take place in the Solar Neighbourhood, but each mechanism in itself turns out to be insufficient to match observational evidences; on the other hand, 
On the galactic disc age-metallicity relation

good agreement is found when both processes are allowed to operate together.

c) Different galactic sub-structures (halo, thick disc, thin disc and bulge), each with its own AMR, might overlap in the Solar Vicinity. Pardi et al. (1994) followed the parallel evolution of halo, thick disc and thin disc with different evolutionary rates, and the resulting mixture of stars shows a scatter in the AMR; but it is lower than the one observed in the Solar Neighbourhood. Most of all, this explanation is not appealing, because only a low contribution from the thick disc and halo population is expected in the Solar Neighbourhood from star counts analysis. In fact, the expected ratio of metal-poor disc stars with respect to other disc stars is about $0.9 \%$ and the ratio of halo/disc $\simeq 1 / 1500(\mathrm{Ng} 1994, \mathrm{Ng}$ et al. 1997), resulting in 2 metal-poor thick disc stars and no halo stars among the Edv93ea F and G stars. The predicted number of stars explains the deficiency of metal-poor stars in Fig. 7. In addition, the expected absence of halo stars is consistent with the disc kinematics of most of the $\mathrm{F}$ and $\mathrm{G}$ stars in the sample. The observed scatter should therefore be intrinsic to the disc and not related to the overlap with other galactic components.

Therefore, new chemical models need to include the possibility of a more complex and composite evolution of the galactic disc than usually conceived; this in turn adds new, uncertain parameters in the discussion of the chemical history of the disc. As correctly underlined by van den Hoek (1997) and van den Hoek \& de Jong (1997), new observational and theoretical constraints are needed to discern between the different processes.

Apart from the problem related with the scatter in the AMR, all chemical models are able to reproduce the main observational constraints of the Solar Neighbourhood, in spite of the different parameterization and assumptions. On the contrary, differences appear when extending the study to the whole disc. In particular, different predictions are given about the evolution of radial abundance gradients with time, although all models are tuned to reproduce the present radial gradients of oxygen and other elements. Tosi (1996) directly compares models from different authors and outlines that in some models the present negative gradient is rapidly established and then remains rather unaltered, in some other models it reaches first a steeper value than currently observed and flattens out afterwards, and yet in other models a positive gradient is initially established, which then decreases and eventually turns to negative values. In fact, the temporal behaviour of the gradient basically depends on the competition between metal enrichment by stellar ejecta and dilution by infalling metal-poor gas, i.e. on the SFR/infall ratio, at different galacto-centric radii and at different ages. Since both the SFR and the infall rate are poorly known due to the uncertainties in the related physical processes, different assumptions can result in different predictions about the evolution of the gradient, though all the models can reproduce the present situation. An observational determination of the radial gradient at different galactic ages would be a strong constraint in discriminating between different chemical models (see Sect. 3.1.1).

To explain the scatter in the local AMR and to describe the evolution of the whole disc in a more consistent way, new codes with more realistic input physics are required. A start is made with the chemo-dynamical models of Steinmetz \&
Table 3. Radial gradient with different age-binning.

\begin{tabular}{lcc}
\hline Age bin (Gyr) & Bin population & $\frac{d[\mathrm{Fe} / \mathrm{H}]}{d R}(\mathrm{dex} / \mathrm{kpc})$ \\
\hline$t<2$ & 18 & $-0.064 \pm 0.013$ \\
$2 \leq t<4$ & 7 & $-0.113 \pm 0.018$ \\
$4 \leq t<6$ & 6 & $-0.118 \pm 0.026$ \\
$t \geq 6$ & 6 & $-0.079 \pm 0.033$ \\
\hline \hline$t<3$ & 21 & $-0.068 \pm 0.012$ \\
$3 \leq t<6$ & 10 & $-0.135 \pm 0.016$ \\
$t \geq 6$ & 6 & $-0.079 \pm 0.033$ \\
\hline \hline$t<4$ & 25 & $-0.086 \pm 0.009$ \\
$t \geq 4$ & 12 & $-0.096 \pm 0.024$ \\
\hline \hline$t<5$ & 29 & $-0.088 \pm 0.009$ \\
$t \geq 5$ & 8 & $-0.061 \pm 0.029$ \\
\hline \hline global & 37 & $-0.085 \pm 0.008$ \\
\hline
\end{tabular}

Müller (1994), Raiteri et al. (1996), Carraro et al. (1997), and Samland et al. (1997). Although arbitrary assumptions are still needed about the SFR, such models can follow infall and gas exchanges among different galactic regions selfconsistently, reducing the number of free parameters and giving a more physical picture of the formation and evolution of the Galaxy.

\section{DISCUSSION}

We now continue with a comparison of the AMRs discussed in the previous section. We first deal with the radial and vertical metallicity gradients observed in the disc, then we proceed with a discussion of the various AMRs and eventually suggest some improvements.

\subsection{Metallicity gradients}

To make a meaningful comparison between AMRs deduced from different samples, they ought to be in or rescaled to the same frame of reference. Positional dependency, due to locally different chemical evolution histories, should be taken into account. But, when correcting for it, one has to consider that the gradients in different age ranges need not be the same, therefore stars or clusters with different ages are not necessarily expected to trace the same metallicity gradient.

\subsubsection{The radial abundance gradient}

The local AMR from Edv93ea, binned with respect to age and radial distance, shows a radial dependence of $[\mathrm{Fe} / \mathrm{H}]$ after a correction of the radial distance for orbital diffusion. The radial gradient seems to be shallower or absent for the oldest stars, but, within the uncertainties inherent in the correction for orbital diffusion and the large scatter in the AMR, the data might also be consistent with a gradient independent of age.

A radial metallicity gradient for open clusters was first found by Janes (1979) and then confirmed by CC94a and Friel (1995). Open clusters are a much better tracer of the 


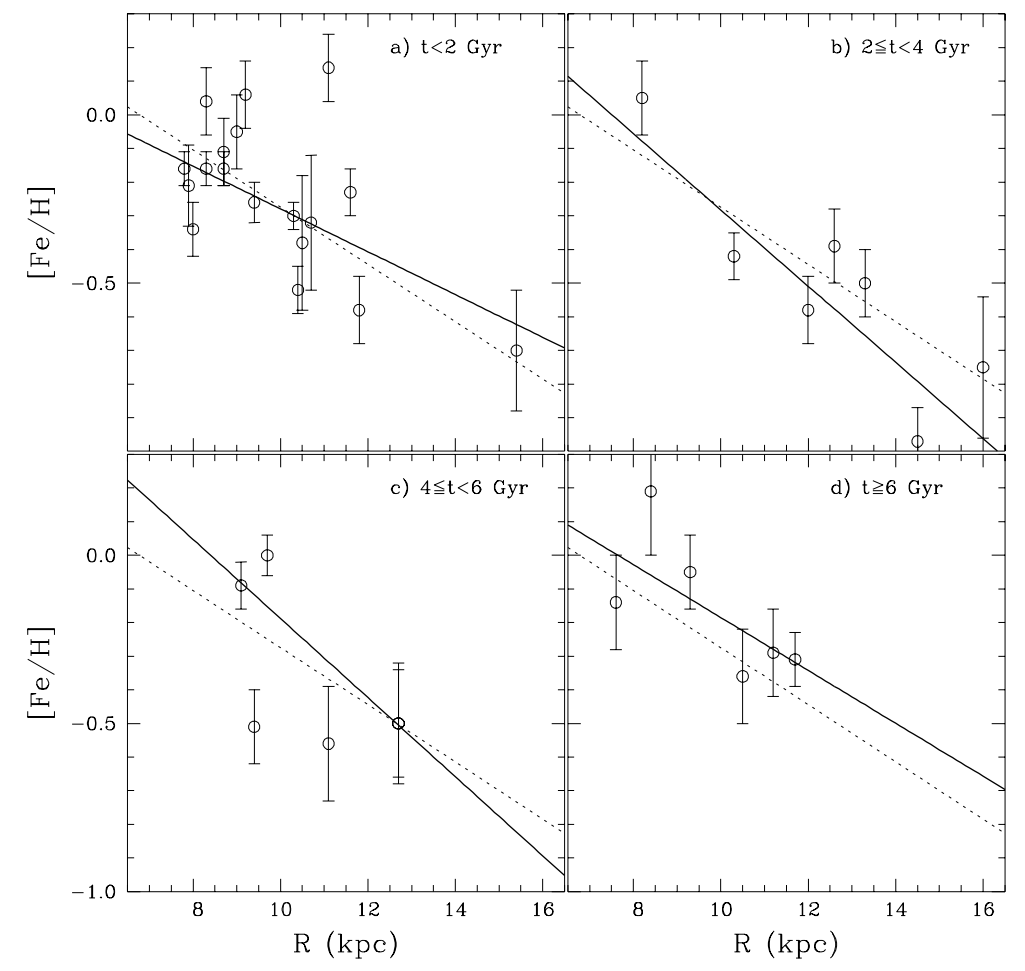

Figure 6. Radial abundance gradients for our sample of open clusters binned in different age ranges. The dashed line (panels $\mathbf{a}-\mathbf{e}$ ) shows the average gradient $\left(-0.09 \mathrm{dex} \mathrm{kpc}^{-1}\right)$ as determined from all the clusters from Table 1. The solid lines in panels $\mathbf{a}-\mathbf{d}$ show the gradients determined for the corresponding age ranges $\left(-0.06,-0.11,-0.12\right.$, and $-0.08 \mathrm{dex} \mathrm{kpc}^{-1}$, respectively).

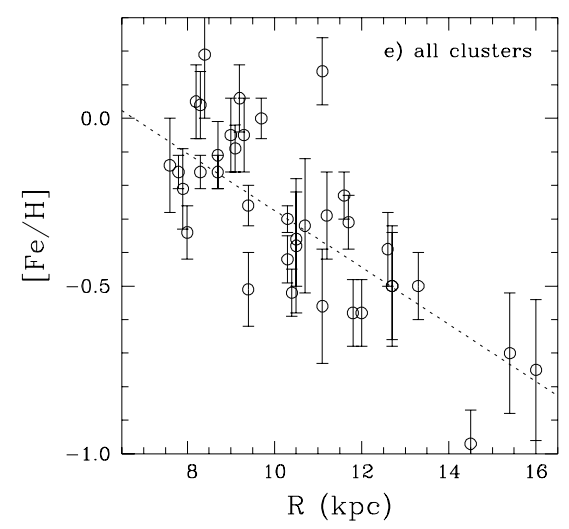

radial gradient than nearby stars, both because they span a larger range of galacto-centric distances and because they are not affected significantly by orbital diffusion. Indeed, orbit calculations have shown that open clusters do not move far away from their birth-places (Carraro \& Chiosi 1994b). A radial gradient is also traced by HII-regions, B-stars, planetary nebulæ (PNæ) and so forth.

B-stars and HII-regions, due to their short lifetimes, are tracers of the present day radial gradient (of oxygen abundance). These two different populations seemed to lead to controversial results: the gradient traced by B-stars was much more shallow (even flat) than the gradient traced by HII-regions (see Prantzos \& Aubert 1995 and references therein). But the latest spectroscopic studies for large homogeneous samples of B-stars indicate a radial gradient $\frac{\mathrm{d}[\mathrm{O} / \mathrm{H}]}{\mathrm{dR}}=-0.07 \mathrm{dex} \mathrm{kpc}^{-1}$ (Smartt \& Rolleston 1997), in agreement with the gradient deduced from HII-regions, so that nowadays the former discrepancy appears to be solved.

As pointed out in Sect. 2.4, the present radial gradient provides a constraint for chemical evolution models, but to discern between different models one needs to know how the gradient evolved in the past. Open clusters are an ideal template for this analysis, since they have well-determined ages, metallicities and galacto-centric distances.

We analyse our homogeneous cluster sample of Table 1 . We divide the sample in age-bins, and for each bin we derive the gradient by means of a weighted least-square fit in the $[\mathrm{Fe} / \mathrm{H}]$ vs $\mathrm{R}$ plane (using a version of the code by Pagel \& Kazlauskas 1992). In the fitting procedure the weights are the errors on $[\mathrm{Fe} / \mathrm{H}]$, also listed in Table 1. A similar analysis was performed by Tosi (1995), but with a smaller sample. Our results for the four age-bins 2 Gyr wide are shown in Fig. 6 and the slopes $\frac{d[\mathrm{Fe} / \mathrm{H}]}{\mathrm{dR}}$ of the gradient for the different age-binning are listed in Table 3. In panels $b-d$ the gradient of panel $a$ is included for comparison.

Note that for the determination of the radial gradient both CC94a and P95ea did not properly consider separate age groups. But the P95ea data set is basically a young sample $(t<1 \mathrm{Gyr})$ and their radial gradient of $-0.07 \mathrm{dex} \mathrm{kpc}^{-1}$ reflects in fact the present day gradient.

Table 3 displays the effects of different age-binning on the derived radial gradients; indicated errors are standard deviations. Before commenting the outcome, a caveat has to be stressed: the number of objects involved in this analysis is relatively low, so statistically the results are not very significant. They show a trend, which requires verification with a larger sample.

By selecting 2-3 Gyr wide age-bins, the results are similar and indicate that the present day gradient is a bit shallower than the past one; the middle epoch seems to display a steepening of the gradient. The choice of wider bins (4 Gyr) basically suggests the same: the present day gradient appears again to be shallower. On the other hand, choosing a bin width of 5 Gyr, we get a different result, probably due to a mixing of the present and middle epoch gradients in the younger age bin. However in this last case the statistics is much poorer, because we are left with only 8 clusters older than 5 Gyr versus 29 in the younger bin.

For the whole sample we derive an average gradient of $-0.09 \mathrm{dex} \mathrm{kpc}^{-1}$, see Fig. 6fe; for any age-bin in Table 3 the gradient is close to this average value, within the errors. In the overall, our sample of clusters seems to show that the radial gradient has not changed much in time, though a hint can be seen for a steeper gradient in the past, at intermediate ages. Therefore, chemical models predicting either a constant gradient or a slightly steeper negative gradient in the past seem to be favoured with respect to models pre- 
On the galactic disc age-metallicity relation

dicting a gradient that settles to the current negative value starting from positive initial values. For a detailed comparison of various chemical models, we refer to Fig. 5 of Tosi (1996) and references quoted therein. Unluckily, due to the large scatter and the small number of clusters in the older age-bins, one cannot actually draw a firm conclusion.

The presence of a metallicity gradient for open clusters has been questioned by Twarog et al. (1997), who find no gradient for the clusters in their sample at galacto-centric radius below $10 \mathrm{kpc}$. However, their Fig. $3 \mathrm{~b}$ displays that the majority of these clusters are located between $7.5-9.5 \mathrm{kpc}$ and have an overall spread of more than 0.2 dex in metallicity. Over a distance range of only $2 \mathrm{kpc}$ our average gradient corresponds to a metallicity difference smaller than 0.2 dex. Therefore, within that dispersion our results are not necessarily at odds with their sample. In addition, Twarog et al.'s claim that open clusters display a step function with a discontinuity at $10 \mathrm{kpc}$ rather than a gradient in metallicity should be verified by separating in age groups. The step function could be a consequence of insufficient discrimination between the contribution from different age groups, because the sample inside $R=10 \mathrm{kpc}$ is heavily weighted toward clusters younger than 1 Gyr, while the outer clusters are predominantly older.

The radial gradient and its age dependence have been studied also by means of PNæ. Maciel \& Köppen (1994) find an oxygen gradient whose present value is in agreement with that of HII-regions. It seems that the gradient has remained roughly constant, or maybe it was slightly shallower in the past. But, unlike open clusters, $\mathrm{PNæ}$ have rather uncertain ages and distances, and their present location might have been spoiled by orbital diffusion. All these factors introduce uncertainties in the derived age dependence of the gradient. Considering the indications from nearby stars, open clusters and $\mathrm{PNæ}$ altogether, the most sensible assumption is that the gradient remained roughly constant in time.

Star counts with the HRD-GST are not sensitive enough to detect a radial gradient within a single-age population. Indeed, a gradient of $-0.21 \mathrm{dex} \mathrm{kpc}^{-1}$ is already induced by differences in scale-lengths among age populations, while within a single-age population the intrinsic gradient of $-0.09 \mathrm{dex} \mathrm{kpc}^{-1}$ is masked out by the adopted extinction along the line of sight.

\subsubsection{The vertical abundance gradient}

The existence of a vertical metallicity gradient among old open clusters is controversial. Friel (1995) and CC94a do not find evidence for such a gradient, whereas P95ea do find a correlation between $[\mathrm{Fe} / \mathrm{H}]$ and vertical position. By binning the data in $z$ they obtained a gradient equal to $-0.34 \mathrm{dex} \mathrm{kpc}^{-1}$. Their sample contains only one cluster above $1 \mathrm{kpc}$, together with 4 clusters above 500 pc. In addition they did not consider NGC 6791, which is high above the plane $(1 \mathrm{kpc})$ and metal-rich. From our sample the resulting slope is shallower: $-0.25 \mathrm{dex} \mathrm{kpc}^{-1}$, not strongly dependent on any particular cluster (Fig. 3, left-bottom panel).

However a caveat is to be stressed, because to estimate the vertical gradient one should disentangle the effects of vertical, radial and age dependence. In principle, one should first bin the sample in homogeneous sub-samples with respect to age and galacto-centric distance, and only then

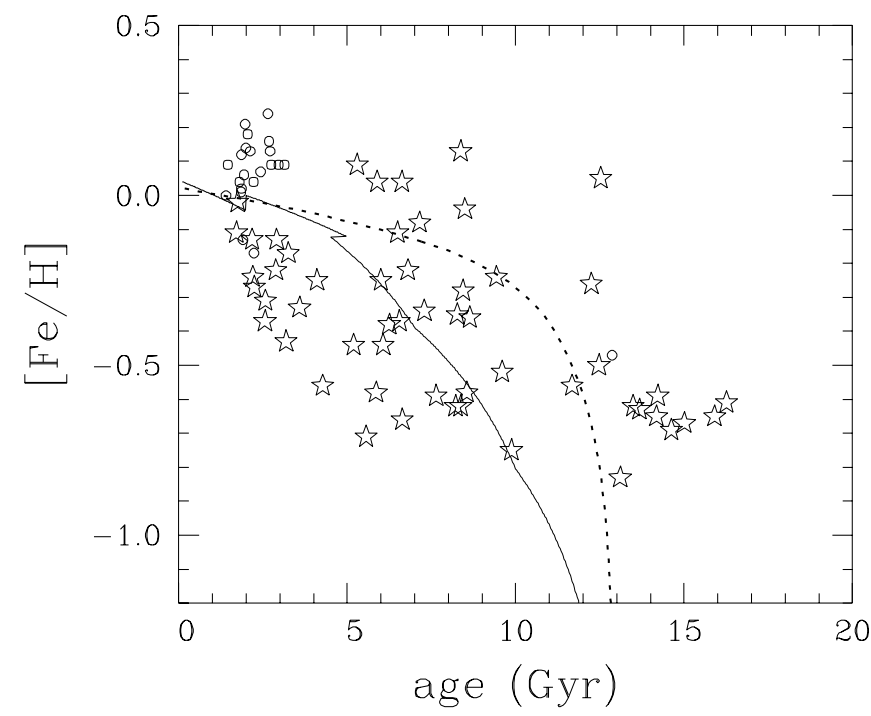

Figure 7. The AMR for stars in the Solar Neighbourhood (open stars and circles, Ng\& Bertelli 1997), see also Fig. 2 for details about the symbol size and uncertainty in the age for these stars; the solid line is the relation obtained for the galactic disc from studies with the HRD-GST (Ng et al. 1996, 1997); the dotted line is the prediction from the chemical evolution model from Portinari et al. (1997), with an adopted age for the disc of 13 Gyr.

analyse the vertical trend. But then, there are not enough clusters in the bins to reliably constrain the vertical gradient. In addition, open clusters form an incomplete sample due to tidal disruption, which is more effective close to the galactic mid-plane. Therefore, clusters at low galactic latitudes are preferentially destroyed, which might introduce a bias in the determination of the vertical gradient.

$\mathrm{Ng}$ et al. (1996) have demonstrated that both the radial and the vertical distribution of open clusters contain information about the scale length and scale height of the galactic stellar populations. In the case of field stars, orbital diffusion is expected to be effective enough to smooth out a vertical metallicity gradient within a single-age population, so that the vertical structure of the disc is dominated by the different scale heights of different age populations. Indeed, in star counts the young, metal-richer stars are confined to regions close to the galactic mid-plane, while the older, metal-poorer stars with a larger scale height dominate at larger vertical distances from the galactic plane. As a consequence, in star counts a vertical metallicity gradient is due to sampling of different age populations rather than reflecting a gradient within a single-age population.

In star counts, radial and vertical abundance gradients are primarily expected due to differences between the scale length and height between different stellar age populations. Since the relative differences in scale length are small with respect to those in scale height, these latter dominate the HRD-GST star counts analysis. A strong vertical abundance gradient in star counts is therefore a hint that insufficient discrimination was made for the different age groups within a data set. A metallicity gradient within an age population is a second order effect.

Almost all open clusters of a given age are found at distances larger than three times the exponential scale length and scale height for the corresponding stellar populations of 


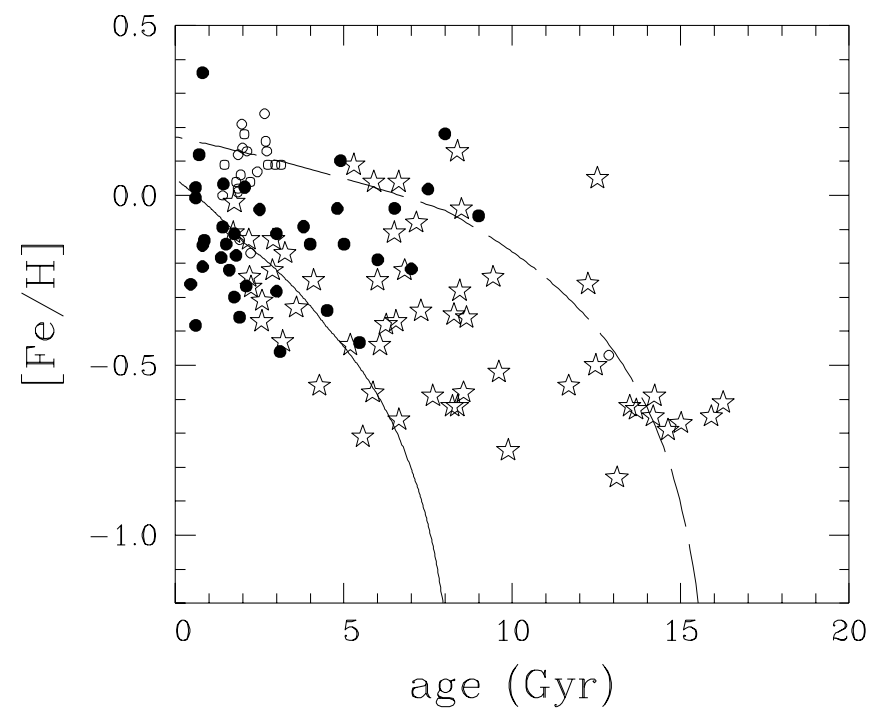

Figure 8. The AMR for open clusters from Table 1 (filled dots) versus the Solar Neighbourhood. The metallicities of the open clusters are corrected according to the global radial gradient: $[\mathrm{Fe} / \mathrm{H}]_{\text {corr }}=[\mathrm{Fe} / \mathrm{H}]_{\text {meas }}-0.09\left(\mathrm{R}_{\odot}-\mathrm{R}\right)(\mathrm{kpc})$. Included in this figure is a suggested improvement for the description of the AMR with two separate components for the HRD-GST. The adopted age for the start of the formation of the 'young' disc component (solid line) is $8.5 \mathrm{Gyr}$ and $16 \mathrm{Gyr}$ for the 'old' disc component (long-dashed line).

the HRD-GST. The vertical gradient due to stellar mixes of different ages, computed at the "transition" region toward

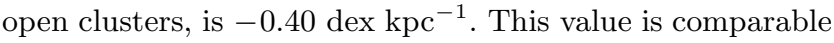
to the one found by P95ea, confirming that their gradient could indeed be due to insufficient discrimination of different age groups.

The orbits of clusters are less affected by orbital diffusion than those of field stars. So, once a vertical gradient for open clusters were established unambiguously, it could provide an important clue about the disc formation history. But, as already stressed, current samples do not give conclusive indications.

\subsection{The galactic disc AMR}

Figure 7 shows various AMRs derived from:

- stars in the Solar Neighbourhood with the ages from NB98 (open circles and open stars);

- the HRD-GST population synthesis analysis for the galactic disc from $\mathrm{Ng}$ et al. (1996, 1997; solid line);

- a chemical evolution model from Portinari et al. (1997; dotted line).

The disc AMR obtained from the HRD-GST is determined from star counts towards the north galactic pole and the galactic centre. No correction for radial or vertical gradients is applied, because star counts are not sensitive to gradients within single-age populations, see Sect. 3.1.1\&3.1.2. For $t<5$ Gyr AMR(HRD-GST:disc) $\simeq \mathrm{AMR}$ (chemics). Between $t=5-13$ Gyr the metallicity of AMR(chemics) is higher than that of AMR(HRD-GST:disc). Indeed, star counts tend to follow the metal-poorer trend, which is more populated and has therefore a larger weight.
Using open clusters to trace the AMR has the main advantage that cluster ages are much more reliably determined than the ages of single field stars, but also the additional problem of the radial and/or vertical dependence of cluster metallicity. Cameron (1985) was the first to derive an AMR from open clusters after correcting for the radial gradient, with the aim at cleaning the data from the space dependence. But a unique radial correction should not be applied recklessly to different age groups, due to the possible agedependence of the radial gradient (Sect. 3.1.1). Unluckily, the age dependence of the radial gradient is poorly constrained and any confident correction for the radial gradient of open clusters is spoiled. Our sample is consistent with a radial gradient which is constant in time, therefore we derive from it the AMR shown in Fig. 8 by correcting for a gradient of $-0.09 \mathrm{dex} \mathrm{kpc}^{-1}$, independent of age. Still, one should bear in mind this uncertainty in the correction, when comparing the AMR of open clusters with the local AMR of nearby stars. In addition, we did not apply any correction for the vertical gradient, since its value or even its existence are not clearly established yet. Figure 8 displays that the AMRs of open clusters and of stars are in good agreement, both showing a similar trend in the scatter. We also notice that both relations show a lack of scattered points in the metal-rich side in the age range 3-5 Gyr and/or an excess of relatively metal-rich objects in the range 5-9 Gyr.

\subsection{The role of the bulge/bar}

The up-turn of the metallicity of the open clusters with possibly a peak near $t \simeq 8 \mathrm{Gyr}$ might be related with the formation of the triaxial bar structure. $\mathrm{Ng}$ et al. (1996) obtained a comparable age and metallicity range $(t=8-9$ Gyr, $\mathrm{Z}=0.005-0.030)$ for this structure. The bar however does not explain the presence of metal-rich stars in the Solar Neighbourhood, because its local density with respect to local disc stars is too low. In addition some of the metal-rich stars are older than the bar.

At $t \simeq 8 \mathrm{Gyr}$, the stars in the Solar Neighbourhood are metal-richer than expected from the AMR(HRD-GST:disc) and AMR(chemics). The calculations from Samland et al. (1997) suggest that this could be due to pre-enrichment with material originating from type II SNæ from the bulge. On the other hand, if the 'bar' structure formed through a merger event some of the metal-richer stars from the inner disc or bulge regions could have migrated near to the Solar Neighbourhood and settled down there. This could explain the presence of rather metal-rich clusters and stars between 5 and 9 Gyr. But suitable dynamical models are required (Mihos \& Hernquist 1996) to explore and check the merger/capture scenario properly against all the observational constraints.

\subsection{Improvements}

The comparison of the AMRs from various methods suggests some improvements.

a) The Solar Neighbourhood: a new sample of stars with spectroscopic metallicities and reliable ages is desirable and better ages are needed for $t>10$ Gyr.

b) Open $\&$ globular clusters: the sample of open clusters 
On the galactic disc age-metallicity relation

should be enlarged to verify the lack of relatively metalrich objects in the age range $3-5 \mathrm{Gyr}$, since the apparent 'U-shape' of the AMR might provide clues about infalling and/or merger events.

The old, metal-rich open clusters and the young, metal-rich globular clusters could indicate when the transition between the two types of clusters actually occurred and provide a clue if this could be initiated by the formation of the galactic bar. The high galactic fore- and background contamination in the colour-magnitude diagrams of the metal-rich globular clusters suggests a combined approach with a galactic population model, to obtain a self-consistent interpretation about the age, metallicity, distance and extinction towards the clusters.

c) Stellar population synthesis: a second, old and metal-rich disc component should be included in the HRD-GST description of the disc (see Fig. 8) and its nature investigated. d) Chemical evolution models: possible pre-enrichment and/or influence of the bulge and of the bar should be considered to explain the metal-rich part of the AMR at old ages. Chemo-dynamical models look promising in this respect. In particular, with the lagrangian approach (Raiteri et al. 1996, Carraro et al. 1997) the behaviour of the gas settling down in the disc, vertical and radial infall, outflows and so on can be followed. In addition, such models are suitable to study merging and capture events.

\section{CONCLUSIONS}

We compared the galactic disc AMRs obtained from four different points of view. Our results are here briefly outlined.

- For the first time a multivariate analysis of a large, homogeneous sample of open clusters was performed and the correlations between cluster parameters were unravelled with an a priori approach.

- We investigated the radial gradient and its time evolution, although a larger sample of open clusters would be required to constrain the past behaviour of the gradient better.

- We showed that an apparent strong vertical gradient in open cluster is likely due to insufficient discrimination between different age groups.

- Considering our improved sample open clusters and a subsample of local stars with reliable ages, both sets of objects trace a similar AMR, whose peculiar shape in the range 3-9 Gyr ago might give clues about past infall or merger events.

\section{ACKNOWLEDGMENTS}

G. Carraro thanks Prof. E. Cappellaro for kind assistance in using SPSS. G. Bertelli is acknowledged for valuable suggestions and discussions. We also thank the anonymous referee for constructive suggestions. This research is supported by the TMR grant ERBFMRX-CT96-0086 from the European Community (Network: Formation and Evolution of Galaxies), by the Italian Ministry of University, Scientific Research and Technology (MURST) and by the Italian Space Agency (ASI). L. Portinari also acknowledges financial support from the Danish Rektorkollegiet.

\section{REFERENCES}

Allen C., Santillan A., 1993, Rev. Mex. Astron. Astrof. 25, 39

Anthony-Twarog B.J., Twarog B.A., 1985, ApJ 291, 595

Aparicio A., Gallart C., 1995, AJ 110, 2105

Aparicio A., Bertelli G., Chiosi C., Garcia-Pelayo J.M., 1990, A\&A 240, 262

Barbaro G., Pigatto L., 1984, A\&A 136, 355

Bertelli G., Bressan A., Chiosi C., Fagotto F., Nasi E., 1994, A\&AS 106, 275

Bertelli G., Bressan A., Chiosi C., Ng Y.K., Ortolani S., 1995, A\&A 301, 381

Bertelli G., Bressan A., Chiosi C., Ng Y.K., 1996, A\&A 310, 115

Bressan A., Chiosi C., Fagotto F., 1994, ApJS 94, 63

Cameron L.M., 1985, A\&A 147, 47

Cannon R.D., 1970, MNRAS 150, 111

Carlberg R.G., Dawson P.C., Hsu T., Vandenberg D.A., 1985, ApJ 294, 674

Carraro G., Bertelli G., Bressan A., Chiosi C., 1993, A\&AS 101, 381

Carraro G., Chiosi C., 1994a, A\&A 287, 761 (CC94a)

Carraro G., Chiosi C., 1994b, A\&A 288, 751

Carraro G., Chiosi C., 1995, in 'The formation of the Milky Way', E.J. Alfaro and A.J. Delgado (eds.), Cambridge University Press, 175

Carraro G., Patat F., 1994, A\&A 289, 297

Carraro G., Patat F., 1995, MNRAS 276, 563

Carraro G., Lia C., Chiosi C., 1997, MNRAS submitted

Charlot S., Worthey G., Bressan A., 1996, ApJ 457, 625

Chiappini C., Matteucci F., Gratton R., 1997, ApJ 477, 765

Chiosi C., Bertelli G., Meylan G., Ortolani S., 1989, A\&A 219, 167

Edvardsson B., Andersen J., Gustafsson B., et al., 1993, A\&A 275, 101 (Edv93ea)

ESA, 1997, The Hipparcos and Tycho Catalogue, ESA SP-1200

Fagotto F., Bressan A., Bertelli G., Chiosi C., 1994, A\&AS 104, 365

Ferrini F., Matteucci F., Pardi M.C., Penco U., 1992, ApJ 387, 138

Ferrini F., Molla M., Pardi M.C., Diaz A.I., 1994, ApJ 427, 745

Friel E.D., 1995, ARA\&A 33, 381

Friel E.D., Janes K.A., 1993, in 'The formation and Evolution of Star Clusters', ASP Conference Series 13, 569

François P., Matteucci F., 1993, A\&A 280, 136 1996, A\&AS 117, 113

Gozzoli E., Tosi M., Marconi G., Bragaglia A., 1996, MNRAS 283,66

von Hippel, T., Gilmore G., Jones D.H.P., 1995, MNRAS 273, L39

van den Hoek L.B., 1997, Ph.D. thesis, University of Amsterdam, the Netherlands

van den Hoek L.B., de Jong T., 1997, A\&A 318, 231

Janes K.A., 1979, ApJS 39, 135

Kaluzny J., 1994, Acta Astron. 44, 247

Maciel W., Köppen J., 1994, A\&A 282, 436

Malinie G., Hartmann D.H., Clayton D.D., Mathews G.J., 1993, ApJ 413, 633

Matteucci F., François P., 1989, MNRAS 239, 885

Mazur B., Krzeminski W., Kaluzny, J., 1995, MNRAS 273, 59

Meusinger H., Reimann H.G., Stecklum B., 1991, A\&A 245, 57

Mihos J.C., Hernquist L., 1996, ApJ 464, 641

Murtagh F., Heck A., 1987, Multivariate Data Analysis, Astrophysics and Space Science Library

Ng Y.K., 1994, Ph.D. thesis, Leiden University, the Netherlands

$\mathrm{Ng}$ Y.K.. Bertelli G., 1998, A\&A 329, 943 (NB98) (astro-ph/9707043

Ng Y.K., Bertelli G., Bressan A., Chiosi C., Lub J., 1995, A\&A 295, 655 (erratum A\&A 301, 318) 
Ng Y.K., Bertelli G., Chiosi C., Bressan A., 1996, A\&A 310, 771 Ng Y.K., Bertelli G., Chiosi C., Bressan A., 1997, A\&A 324, 65 Nie, N.H., Hadlai Hull, C., Jenkins, J.G. et al., 1975, SPSS, Statistical Package for the Social Science, 2nd edition, McGraw-Hill, New York

Pardi M.C., Ferrini F., Matteucci F., 1994, ApJ 444, 207

Pagel B.E.J., Kazlauskas A., 1992, MNRAS 256, 49

Pagel B.E.J., Tautvaisiene G., 1995, MNRAS 276, 505

Phelps R.L. Janes K.A., Montgomery K.A., 1994, AJ 107, 1079

helps R.L., 1997, ApJ 483, 826

Piatti A.E., Claria J.J., Abadi M.G., 1995, AJ 110, 2813 (P95ea)

Portinari L., Bressan A.G., Chiosi C., 1997, A\&A submitted

Prantzos N., Aubert O., 1995, A\&A 302, 69

Pilyugin L.S., Edmunds M.G., 1996, A\&A 313, 783

Raiteri C.M., Villata M., Navarro J.F., 1996, A\&A 315, 105

Samland M., Hensler G., Theis Ch., 1997, ApJ 476, 544

Smartt S.J., Rolleston W.R.J., 1997, ApJ 481, L47

Steinmetz M., Müller E., 1994, A\&A 281, L97

Timmes F.X., Woosley S.E., Weaver T.A., 1995, ApJS 98,617

Tosi M., 1988, A\&A 197, 33

Tosi M., 1995, in 'The formation of the Milky Way', E.J. Alfaro and A.J. Delgado (eds.), Cambridge University Press, 153

Tosi M., 1996, in 'From stars to galaxies', C. Leitherer, U. Fritzevon Alvensleben and J. Huchra (eds.), ASP Conference series vol. 98,299

Tosi M., Greggio L., Marconi G., Focardi P., 1991, AJ 102, 951

Twarog B.A., 1980, ApJ 242, 242

Twarog B.A., Ashman K.M., Anthony-Twarog B.J., 1997, AJ 114, 2556 (astro-ph/9709122)

Vallenari A., Chiosi C., Bertelli G., Meylan G., Ortolani S., 1992, AJ 104, 1100

Vandenberg D.A., 1983, ApJS 51, 29

Vandenberg D.A., 1985, ApJS 58, 711

van den Bergh S., McClure R.D., 1980, A\&A 80, 360

Wheeler J.C., Sneden C., Truran J.W.jr., 1989, ARA\&A 27, 289

Wielen R., 1977, A\&A 60, 263

Wielen R., Dettbarn C., Fuchs B., et al., 1992, IAU Symp 149, p. 81

Wielen R., Fuchs B., Dettbarn C., 1996, A\&A 314, 438 